\title{
EFFECTS OF VARIATIONS IN THE AVAILABLE BANDWIDTH ON THE PERFORMANCE OF THE GFR SERVICE
}

\author{
Norbert Vicari \\ University of Würzburg, Computer Science, Am Hubland, D - 97074 Würzburg, Germany \\ e-mail:vicari@informatik.uni-wuerzburg.de
}

\begin{abstract}
The Guaranteed Frame Rate service category is currently under discussion for incorporation into the specification documents of the ATM-Forum. The concept of the GFR service is to provide a minimum service guarantee to classical besteffort services, taking into account the frame-based nature of todays data-traffic. In this paper we present a discrete-time analysis of the GFR service and discuss the effects of variations of the bandwidth available for the GFR service. The presented method can be applied to dimension the thresholds of the algorithms used to enforce the guaranteed service.
\end{abstract}

Keywords: GFR, ATM, Performance, Discrete Time Analysis, F-GCRA

\section{INTRODUCTION}

The ATM-Forum recently introduced the Guaranteed Frame Rate (GFR) service category [6], which is currently considered to be incorporated into the ATM-forum specification [3]. This new service category is motivated by several intentions. Today most applications are not equipped to select the proper traffic parameters required to establish ATM connections. Thus, choosing CBR or VBR service categories will fail either by causing inefficiency by overestimating required resources or not being able to give any QoS guarantees. The ABR service category is regarded to be too complex to be implemented in the majority of systems.

Transferring data traffic with the best-effort service category UBR would avoid the problem of estimating traffic descriptors, but will also give no QoS guarantees at all. Worse, the throughput seen at higher protocol layers is severely reduced. Most of todays applications utilize the Transmission Control Protocol/Internet Protocol (TCP/IP) for transferring data in frame based 
structures. When transmitting these frames over an ATM network the data is fragmented into cells. The loss of a single cell will cause an irrecoverable damage to the whole frame and induce retransmission. To cope with these problems, the GFR service category provides the user with a Minimum Cell Rate (MCR) guarantee under the assumption of a given Maximum Frame Size (MFS) and Maximum Burst Size (MBS). The user is allowed to send traffic in excess of the negotiated parameters, but this traffic will only be delivered within the limits of available resources.

The resources available for the GFR service alternate in different timescales. Fast fluctuations are caused by higher priority VBR connections, while, the average share of bandwidth for the GFR service is mainly influenced by the establishment and release of other ATM connections. In [9] we considered constant available bandwidth reflecting the steady-state effects of long-term fluctuations of the availability of resources. In this paper we will also take short-term variations of the resource availability into account.

The paper is organized as follows: in Section 2 an overview of the GFR service category and its key components are given. Section 3 describes the modeling and analysis of the system. Numerical examples derived with the presented analysis method are provided in Section 4. The paper is concluded with a summary.

\section{THE GFR SERVICE CATEGORY}

Motivated by the needs of a guaranteed minimum bandwidth for besteffort ATM connections the introduction of a MCR for the UBR service category was suggested [5]. This considerations resulted in the definition of the so called UBR+ service category [4]. The main idea of this service category was to preserve the best-effort properties of the UBR service category while adding the guarantee of a minimum bandwidth. The newer specification of this service category [6] names the service GFR, which reflects the approach of taking frames into account for the minimum guaranteed bandwidth.

In comparison to $\mathrm{ABR}$, which also provides a guaranteed best-effort service, GFR is easier to implement and does not add a new flow control scheme. Thus, implementation of GFR in adapter cards and network nodes is expected to be faster and cheaper. Further, the coupling of different flow control schemes - like TCP over ABR - may lead to unpredictable and unintended complications.

The VBR.3 service category [1] also allows the user to send traffic in excess to the traffic contract, but in comparison to the GFR service the traffic is not regarded as flow of frames. Since, most currently available applications 
use the TCP/IP protocol, data is organized in frames and has to be fragmented into cells for transport over an ATM net. Thus, random loss of a single cell leads to corruption of the whole frame and reduces the goodput of the transmission. Discarding whole frames that are not eligible for guaranteed transmission increases the goodput of the net.

The GFR service is intended to support non-real-time traffic and requires the data being organized in frames which can be delineated at the ATM layer. The user is provided with a MCR guarantee when transmitting frames that do not exceed the MFS in a burst that does not exceed the MBS. Frames sent in excess to this parameters will be delivered only within the limits of available resources.

The GFR service [2] provides a guarantee to deliver complete unmarked frames that are conforming and eligible. A frame is defined to be conforming if the CLP bit of all cells of a frame has the same value as the CLP bit of the first cell of the frame, the number of cells on the frame is less than MFS and the rate of the cells conforms to the parameter Peak Cell Rate (PCR), which is monitored with a conventional Generic Cell Rate Algorithm (GCRA). The eligibility of frames is defined with the Frame-Based-GCRA (F-GCRA), which controls if the rate of the cells of a frame is less than MCR and the length of burst is less than MBS. In order to stick to the frame-based approach all cells of a frame are valued identically to the first cell of a frame.In order to provide the above defined service guarantee the network has to discriminate between eligible and non-eligible frames when transmitting data with the GFR service. Therefor, a queuing discipline with two thresholds is applied to guarantee the transmission of eligible frames while providing best effort service for the remaining frames.

In the following we will review the queuing discipline applied for the GFR service after introducing the exact functionality of the F-GCRA.

\subsection{Frame-Based Generic Cell Rate Algorithm: F-GCRA}

Figure 1 shows how the $F-G C R A(I, L)$ algorithm decides if an arriving frame is eligible or not according to a given increment parameter $I$ and a limit parameter $L$. The only basic difference of $F-G C R A(I, L)$ to a $G C R A(I, L)-$ as formerly been defined by the ATM traffic management standard [1] - is that only the first cell of a frame is checked according to the GCRA while effectively tagging the whole frame as eligible or non-eligible. Frame cells arriving later only update the F-GCRA state if the first cell was eligible but they are never checked against the limit parameter $L$.

The simplified F-GCRA algorithm above assumes that the frame stream is already conforming to the $P C R$ with frame sizes no bigger than $M F S$. For the 


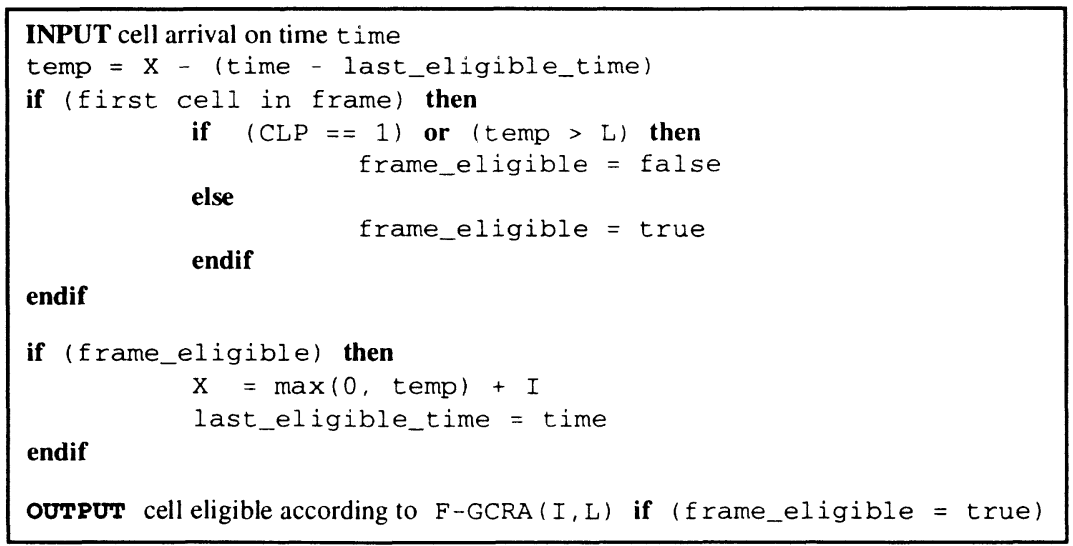

Figure 1: F-GCRA $(I, L)$ implemented as virtual scheduling algorithm.

treatment of non-conforming frames no binding rule exists. However, it is very common practice to regard a non-conforming frame as a strict violation of the traffic contract with the immediate consequence of complete frame rejection. Hence, for the addressed GFR performance evaluation an inclusion of further details in the algorithm is neither necessary nor sensible. Figure 2 shows an snap-shot of the F-GCRA state for an example scenario with three consecutively arriving frames.

The first two frames are declared to be eligible according to $F-G C R A(I, L)$ as the F-GCRA state upon arrival of the first cell does not exceed the limit parameter $L$. For each arriving cell of these two frames the F-GCRA state is increased by the increment parameter $I$. The third frame is declared non-eligible and the F-GCRA is never increased upon arrival of any frame cell.

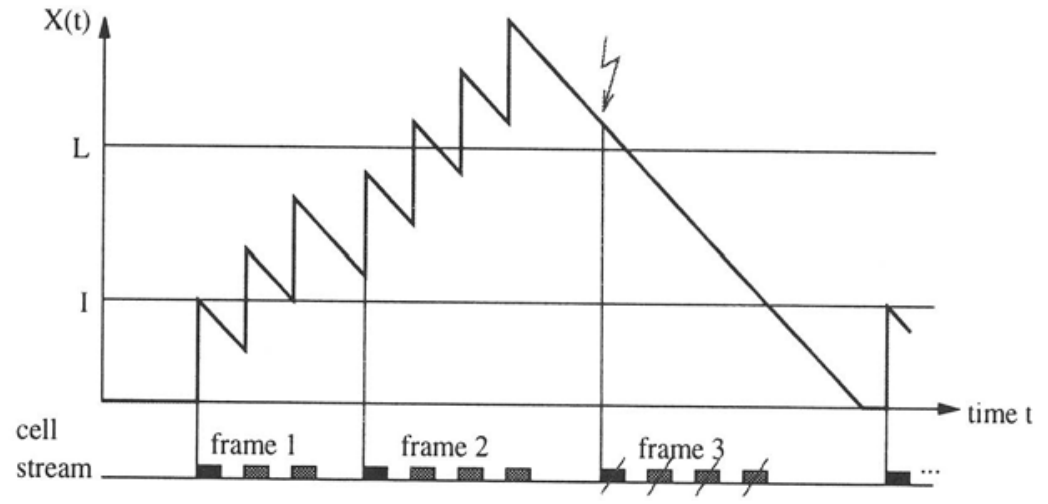

Figure 2: F-GCRA state diagram with three arriving frames. 


\subsection{Queuing Discipline}

After classifying the frames with help of the F-GCRA the network node has to transmit the frames eligible for service guarantee with low loss probability. If additional resources are available on the transmission link, frames sent in excess to the traffic contract should be also transferred. Naturally these frames will suffer a higher loss rate than frames with guaranteed service. Cells which could not be transferred immediately are stored in a buffer of size $Q \_M A X$. When a cell of a frame - eligible or non-eligible - could not be stored in the buffer, this cell and all subsequent cells of this frame are discarded since it is assumed that the loss of a single cell of a frame leads to the retransmission of the whole frame.

To discern eligible and non-eligible frames two threshold values are introduced. The Low Buffer Occupancy $(L B O)$ value indicates the limit for the acceptance of non-eligible frames. That is, if at the time instant of the arrival of the first cell of a non-eligible frame at least $L B O$ cells are waiting in the buffer the whole frame is discarded. Analogously the High Buffer Occupancy $(H B O)$ value defines the limit for the acceptance of eligible frames. Once the first cell of a frame is accepted the subsequent cells of this frame could be only discarded due to buffer overflow. In order to investigate the influence of different values of $\angle B O$ and $H B O$ we model the correlated system of F-GCRA and queue.

\section{MODELING AND ANALYSIS}

In the following, a model and its corresponding discrete-time analysis of the GFR service are presented. The issue of conforming/non-conforming frames will be neglected, that is, all arriving frames are considered conforming. After a description of the frame arrival process we will evaluate the system state of the coupled model of F-GCRA and transmission line as shown in Figure 3.

For the analysis of the model we will describe the system state by a two dimensional random variable $\left(X_{f}, X_{q}\right)$. The first dimension represents the

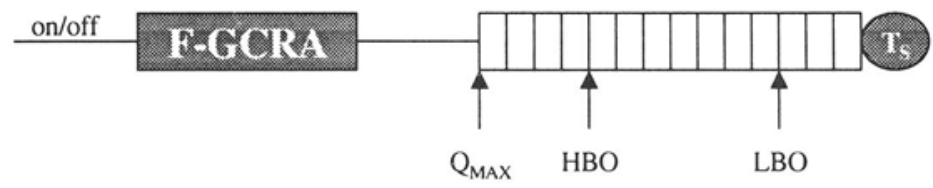

Figure 3: The basic GFR model 
leaky bucket counter of the F-GCRA and the second dimension represents the time required to transmit the cells waiting in the buffer.

All random variables to describe the system state are measured in multiples of the duration of a cell transmission at PCR. The capacity of the transmission link is denoted by the RV $T_{S}$, that is, the duration of the transmission of a cell takes $T_{S}$ time units. Then, the capacity $\mathrm{QL}$ of the buffer including the transmission unit can be approximated using the average cell transmission duration $\bar{T}_{S}$ :

$$
Q L=\left(Q_{M A X}+1\right) \overline{T_{S}}-1 .
$$

Analogously the limits for the acceptance of eligible and non-eligible frames HBL and $\mathrm{LBL}$ - expressed in time-slots - are defined:

$$
\begin{aligned}
& L B L=(L B O+1) \overline{T_{S}}-1, \\
& H B L=(H B O+1) \overline{T_{S}}-1 .
\end{aligned}
$$

An analysis of the F-GCRA for determining the parameters MCR and MBS can be found in [8].

\subsection{Modeling the Arrival Process}

The functionality of the GFR service is based on the organization of data in frames. Most of the currently used protocols e.g. TCP/IP transport data in frames that have to be split up in several cells for transport over an ATM network. This sort of traffic can be modeled by the class of on/off-processes. An on-state represents the transmission of a frame, while the off-state represents the time between the frames. In our analysis we consider an on/off source as depicted in Figure 4.

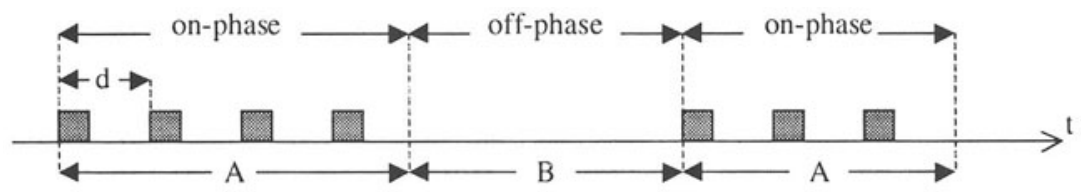

Figure 4: On/off source

The duration of the on- and off-phases are distributed according to discrete general and independent distributions. At the beginning of a on-phase a cells arrives immediately. During an on-phase cells arrive in intervals of $d$ time units, which correspond to the transmission time of a cell $1 / P C R$. The end of an on-phase is not generally synchronized to a cell arrival.The duration of the on-phase is denoted by the random variable $\mathrm{A}$ and the duration of the offphase by B, respectively. 


\subsection{System State Evolution}

The random variable $\left(X_{f}^{o n}, X_{q}^{o n}\right)$ describes the system state at the beginning of an on-phase. Tracing every cell arrival the system state after the arrival of the last cell of a frame is iteratively derived. Taking into account the time remaining in the on-phase the system state at the beginning of the offphase $\left(X_{f}^{\text {off }}, X_{q}^{\text {off }}\right)$ is given. Since no cell arrivals occur during the off-phase the system state is decreased until the beginning of the next on-phase is reached.

\section{Transition Off-Phase to On-Phase}

The state of the F-GCRA is reduced during the off-phase by $B$ units, since no cell arrival occurs. Before the arrival of the next frame the state of the FGCRA computes as follows:

$$
X_{f}^{o n}=\max \left(X_{f}^{o f f}-B, 0\right) \text {. }
$$

Analogously at most B units of virtual work could be served at PCR during the off-phase, thus the state of the queue at the beginning of the on-phase is given by the following equation:

$$
X_{q}^{o n}=\max \left(X_{q}^{o f f}-B, 0\right) .
$$

\section{Transition On-Phase to Off-Phase}

During the on-phase cell arrivals occur starting with the first time slot and continuing every $d$ time slots, c.f. Figure 4 . The system state is denoted recursively, and the system is observed at the end of time slot $k$.

At the beginning of the on-phase the F-GCRA takes the following state:

$$
X_{f}^{o n, 0}=X_{f}^{o n} \text {. }
$$

The state of the F-GCRA at the end of an on-phase of $A$ time units is computed recursively as follows:

$$
X_{f}^{o n, A}=\left\{\begin{array}{l}
X_{f}^{o n, A-1}-1+I, \quad \text { if } X_{f}^{o n, 0} \leq L \text { and cell arrival } \\
\max \left(0, X_{f}^{o n, A-1}-1\right), \text { else }
\end{array} .\right.
$$

For the derivation of the buffer state we discern the arrival of the first and the subsequent cells of a frame. The state of the buffer before the arrival of the first cell is given by:

$$
X_{q}^{o n, 0}=X_{q}^{o n} .
$$


Upon the arrival of the first cell of a frame the frame acceptance is decided. If the state of the buffer at most $L B L$ all frames are accepted. Eligible frames are accepted even if the state of the buffer is higher then $L B L$ but at most $H B L$. If a cell could not be accepted all remaining cells of the frame are also discarded. We denote this changing the value of the RV DF from 0 to 1 .

$$
X_{q}^{o n, 1}=\left\{\begin{array}{ll}
X_{q}^{o n, 0}-1+T_{S}, & \text { if } X_{f}^{o n, 0} \leq L \text { and }\left(X_{q}^{o n, 0} \leq H B L-T_{S}\right) \\
X_{q}^{o n, 0}-1+T_{S}, & \text { if } X_{f}^{o n, 0}>L \text { and }\left(X_{q}^{o n, 0} \leq L B L-T_{S}\right) \\
\max \left(0, X_{q}^{o n, 0}-1\right), & \text { else }
\end{array} .\right.
$$

The remaining cells of a frame are discarded only if either the buffer is occupied or some cells of the frame have already been discarded. Thus the state of the buffer is recursively computed as follows:

$$
X_{q}^{o n, A}=\left\{\begin{array}{ll}
X_{q}^{o n, A-1}-1+T_{S}, & \text { if } X_{q}^{o n, A-1} \leq Q L-T_{S} \\
& \text { and } D F=0 \text { and cell arrival } \\
\max \left(0, X_{q}^{o n, A-1}-1\right), & \text { if } D F=1 \text { and cell arrival } \\
\max \left(0, X_{q}^{o n, A-1}-1\right), & \text { if not cell arrival } \\
\max \left(0, X_{q}^{o n, A-1}-1\right), & \text { else }
\end{array} .\right.
$$

The 'else' branch of equ. (8) and (9) indicates the first discarding of a cell and thus initiates a change of the value of $D F$ from 0 to 1 .

\subsection{Discrete-Time Analysis}

In order to obtain the frameloss probability the probability mass function describing the system in equilibrium state are derived. Due to space limitations the probability mass function description corresponding to the RV description of equ. (3) to equ. (9) is omitted here, but can be found in an extended version of this report [10]. In difference to the common discrete time analysis approach the state space is divided in 4 semi-distributions to realize the memory of the system regarding frame loss.

\section{NUMERICAL EXAMPLES}

For the presentation and discussion of numerical examples we will refer to the following basic parameter set unless otherwise expressed. 
The length of the on- and off-phase of the considered traffic stream is distributed geometrically. In order to represent a cell indicating the beginning and the end of a frame, the minimum length of an on-phase is set to 2 . The system load is chosen to be $25 \%$ of the $P C R$, for example for $d=1$ - the cells of a frame are sent back to back - the on-phase is set to be 10 slots and the offphase to 30 slots in average. For other values of $d$ the duration of the on- and off-phase is adopted accordingly.

The MCR of the F-GCRA is set to $20 \%$ of the link cell rate, that is $I=5$. With a limit $L=100$ for cells sent back to back we obtain a ratio of $74 \%$ eligible frames. For varying burstiness of the traffic stream the limit of the FGCRA is adopted accordingly. The required parameters could be easily computed with the analysis method presented in [8].

The transmission-time distribution $t_{\mathrm{s}}$ is chosen to adopt only two values one below and one above the expectation - to reduce the computational complexity of the analysis. The values are adjusted accordingly to fit the selected expectation $T_{S}$ and the coefficient of variation $c$.

\subsection{GFR Functionality}

First we look at the queuing behavior of a single link carrying GFR-traffic. In order to reflect the best-effort characteristics of the GFR service the bandwidth available for the connection is set to $1 / 3$ of the $P C R$, which should fulfill the bandwidth requirement of the reference traffic stream. The coefficient of variation of the available bandwidth is $c=1.0$. Figure 5 shows the conditional frame loss probability for eligible and non-eligible frames in dependence of the $\angle B O$. Lower $L B O$ values mean that even in the case of low buffer occupancy non-eligible frames are discarded. Thus, eligible frames are served with higher reliability and suffer lower loss. But on the other hand the transmission buffer for the whole traffic stream is reduced by preferential treat-

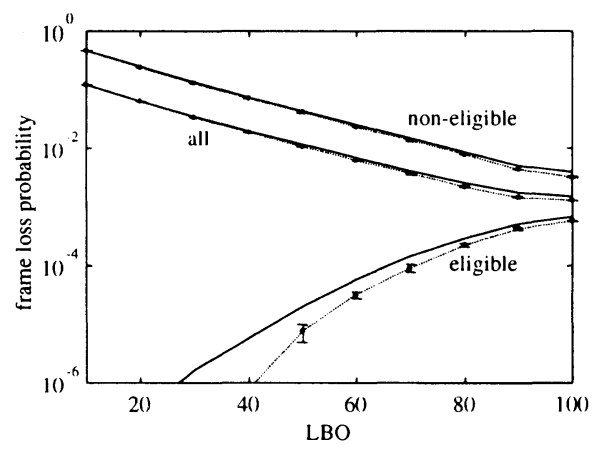

Figure 5: Functionality of the GFR service. 
ment of the eligible frames, which leads to a reduction of the total throughput of the system. Thus, the functionality of the GFR service depends on the proper dimensioning of the $L B O$.

To demonstrate the approximation accuracy of the analysis some simulation results are included in the graphic. Generally the simulation results depicted with dashed lines and $95 \%$ confidence interval - show good accordance with the results of the analysis. Since, the approximation in the analysis depends on the length of the queue the results are more accurate for higher share of the queue between eligible and non-eligible frames. Reducing the variance of the transmission time distribution also increases the accuracy of the analysis. For deterministic transmission time the analysis even is exactly.

\subsection{Factors Influencing the Performance of GFR}

To assess the effects of long-term and short-term fluctuations of the available bandwidth the blocking probability of eligible frames for different amounts of available bandwidth is depicted in Figure 6 (left side). In our example the traffic stream causes a load of $25 \%$. The ratio of eligible frames is $74 \%$. Thus, the system is capable to transfer eligible frames with a available bandwidth of $20 \%\left(\mathrm{~T}_{\mathrm{S}}=5\right)$ of the PCR if the $L B O$ is dimensioned properly. If higher capacity is available for the GFR service, the $L B O$ can be chosen higher to obtain the same maximum blocking probability for eligible frames and to increase the overall throughput, cf. Figure 5.

In most cases the short-term variation of the available bandwidth increases the blocking probability only by a negligible amount, since, the buffer available for eligible frames is sufficient to compensate short-term variations of the transmission time. If the average available bandwidth changes the effects are by far stronger and require a modification of the threshold $L B O$ to guarantee the service for eligible frames.
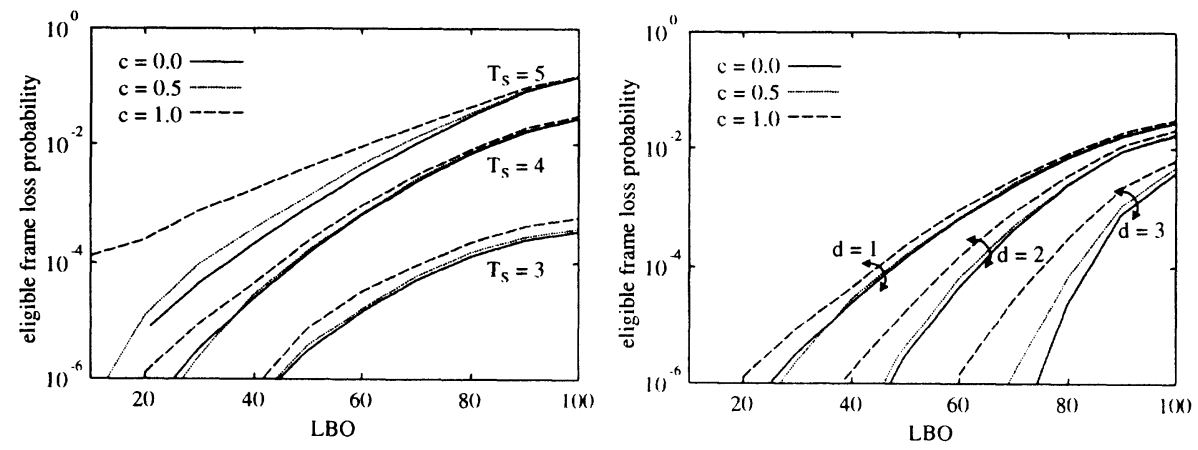

Figure 6: Influence of the available capacity and the traffic-streams burstiness. 
To evaluate the impact of the burstiness of the traffic stream on the GFR service the following system configuration is used: the bandwidth available for the GFR service is set to $25 \%$ of the PCR, that is equal to the minimum bandwidth required by the traffic source. The cells in a frame are spaced by $d$ time slots, cf. Section 3.1. The duration of the on- and off-phase are adjusted accordingly to ensure a traffic load of $25 \%$. The parameters of the F-GCRA are adjusted to classify $74 \%$ of the frames as eligible, utilizing the analysis introduced in [8]. As shown in Figure 6 (right side) the blocking probability of eligible frames increases with increasing burstiness. Short-term variation of the available bandwidth compared with the traffic streams burstiness has minor impacts on the performance. Again, to obtain an identical maximum blocking probability the parameter $L B O$ has to be adjusted accordingly.

The numerical examples show that the dimensioning of the parameters for the GFR service depends heavily on the traffic characteristics and average amount of available bandwidth. Short-term variations of the available bandwidth do not reduce the quality of the GFR service significantly. The Information about the traffic characteristics could be gained from the attributes of a GFR-connection [7] and taken into account. If sufficient bandwidth is available the - with regard to the service guarantee - highest possible $L B O$ value should be chosen to obtain a high throughput of eligible- and also non-eligible frames. But if the only the minimum bandwidth MCR for the GFR-connection is available the $L B O$ has to be chosen more restrictively. A restrictive selection of the $L B O$ will fulfill the service requirements in any case, but not the expectations to a best-effort service class.

\section{SUMMARY}

In this paper we presented a discrete-time analysis of the GFR service category, which is currently defined by the ATM Forum. The both key components of the GFR service - the F-GCRA algorithm and the transmission queue - are described and modeled. While the F-GCRA discriminates eligible and non-eligible frames the buffer discipline ensures the service quality guaranteed by the GFR service category. To model the frame-based cell arrivals a on/ off-process with generally distributed on- and of-phase was chosen. Since the state of the F-GCRA and the queue are correlated, a two-dimensional discrete-time analysis approach is applied.

The average available bandwidth and the traffic characteristic are identified in the numerical examples as the determining factors for the performance

of the GFR service, while short-term variations of the available bandwidth can be neglected. The results indicate a discrepancy in the dimensioning of 
the parameters of the queuing discipline. A restrictive selection of the relevant parameter $L B O$ ensures a transmission of eligible frames with the guaranteed service quality, but the best-effort spirit of the GFR service is given away. Thus, a dimensioning of the queuing discipline of the transmission buffer in dependence of the currently available bandwidth is an interesting approach to guarantee the service and to preserve the best-effort characteristic of the GFR service.

\section{Acknowledgment}

The author would like to thank Robert Schedel for his programming efforts during the course of this work. Further the financial support of the Deutsche Telekom AG (Technologiezentrum Darmstadt) is appreciated.

\section{References}

[1] Traffic Management Specification Version 4.0. The ATM Forum Technical Committee, April 1996.

[2] Traffic Management Baseline Text Document, BTD-TM-01-02. The ATM Forum Technical Committee, July 1998.

[3] N. Giroux and J. Kenney. Proposed Additions to the Traffic Management 4.1 Draft Specification. The ATM Forum Technical Committee 98-0626, October 1998.

[4] R. Guerin and J. Heinanen. UBR+ Service Category Definition. The ATM Forum Technical Committee 96-1598, December 1996.

[5] J. Heinanen. MCR for UBR. The ATM Forum Technical Committee 960362, April 1996.

[6] S. Jagannath, N. Yin, J. B. Kenney, J. Heinanen, J. Axell, K. K. Ramakrishnan. Modified Text for Guaranteed Frame Rate Service Definition. The ATM Forum Technical Committee 97-0833, December 1997.

[7] G. Koleyni. Updating Table 2-1. The ATM Forum Technical Committee 98-0450, July 1998.

[8] M. Ritter. Discrete Time Modeling of the Frame-Based Generic Cell Rate Algorithm. Univ. of Würzburg, Research Report, No. 190, January 1998.

[9] N. Vicari and R: Schedel: Performance of the GFR-Service with Constant Available Bandwidth. Proceedings of the IEEE Infocom'99, March 1999, New York, USA.

[10] N. Vicari: Effects of Variations in the Available Bandwidth on the Performance of the GFR Service. Univ. of Würzburg, Research Report, No. 214, October 1998.

ftp://www-info3.informatik.uni-wuerzburg.de/pub/TR/tr214.pdf. 